Aus der chirurgischen Universitätsklinik zu Frankfurt a. M. (Direktor: Professor Dr. V. Schmieden.)

\title{
Zur Frage der Entstehung und Behandlung der subkutanen Ruptur der langen Bicepssehne.
}

Ion Dr. Gustav Rosenburg, Assistent der Klinik.

Unter der Bezeichnung "Bicepsruptur" werden dic Verlctzungen im Bcreiche des muskulären und schnıgen Teiles des Muskels zusammengefaßt. Meist handelt es sich um V'erände. rungen im Bereiche des langen Kopfes des Muskels, und zwar um Abrisse oder Einrisse an der proximalen Schne des langen Kopfes. Seltenor sind die Abrisse der gemeinsamen distalen Sehne der zwci, oder in scltenen Fällen auch drei Köpfe des Muskels. Zerreißungen im Bereiche der Muskelsubstanz, die in 2 Fällen auch der: inneren Kopf umfaßłten, sind in der Regel die Folgo: einer direkten (iewalteinwirkung auf den Muskel. Über die sub. kutane Bicepsruptur durch dirckte Gewalt hat Hennemann an Hand von 7 lällen in einer Dissertation berichtet. Die Biceps. ruptur durch indirekte (iewalt entsteht viel häufiger als die durch direktc Gewalt. L,iniger hat hei seiner Tätigkeit als Gutachter weit über roo Fälle von Einrissen und Abrissen der Biceps. sehne durch indirektc Gewalt gesehen. Diese lälle sind in ge. wisser Bezichung von denen z.u unterscheiden, die bisher veröffent. licht worden sind, denn es handelt sich meist um Veränderungen im Berciche der proximalen Sehne des langen Bicepkopfes, die von dem Betroffenen sclbst gar nicht bemerkt wurden, während bei den in der Literatur veröffentlichten Fällen die Anzcichen einer frischen Verletzung oder wenigstens einer schweren Schädigunģ vorlagen.

Bei einem AbriB der distalen Sehne des Biceps orler einer Ruptur im Bcreiche der Muskelsubstanz ist die operative Anheftung ar det Tuberositas radii bzw. eine Vereinigung der Stümpfe 
durch die Naht dringend geboten. Die Ergebnisse nach chirurgischem Vorgehen in diesen Fällen sind nach dem Urteil aller Autoren ausgezcichnet. Dic Fälle, in denen die Operation von dem Betroffenen abgelehnt wurde, zeigten ein sehr schlechtes funktionelles Endresultat, wie z. B. der Fall Schülein. Nicht so klar liegen die Verhältnisse bei einer Ruptur im proximalen sehnigen Teil des langen Bicepskopfes. Die normale Anatomie des Biceps sei kurz angeführt.

Der lange Kopf des Biceps nimmt scinen Lirsprung an der Tuberositas supraglenoidalis, von wo dic Sehne durch das Schultergelenk und den Sulcus intertubercularis zieht. Bis unterhalb dieses Sulcus ist sic von einer Ausstülpung der Gelenkkapsel umgeben, der Vagina mucosa intcrtubercularis, dic von manchen Anatomen auch als Schnenscheide für die lange Bicepssehne bezeichnet wird (Frohse und Fracnkel). Eirst am unteren Rande bzw. am Oberarmrande des quer über den Arm verlaufenden Pectoralis major beginnt der muskuläre Teil des Biceps. In der Mitte des Oberarmes, in der Nähe der Tuberositas deltoidea vereinigt sich der jetzt muskuläre Teil des langen Kopfes mit dem kurzen Bicepskopf, der am Processus coracoideus entspringt und mehr medial am Oberarm verläuft. Der gemeinsame Muskelbauch inscriert in der Hauptsache mit starker Schne an der Tuberositas radii, während cin geringercr Teil, zum Caput breve gchörig, in den Lacertus fibrosus übergeht und nach der ulnaren Seite des Unterarmes zieht.

Die Funktionen des Muskels ergeben sich aus seinem Verlaut. Der Biceps ist nicht nur Beuger des Armes, sondern auch kräftiger Supinator, was durch den schrägen Verlauf zum Radius verständlich wird. In Supinationsstellung kann sich der Biceps am meisten zusammenziehen und leistet auch die meiste Arbcit. Die Wirkung auf dic Bewegungen des Armes in der Schulter sind nach B ra us um $1 / 3$ geringer als auf Flexion und Supination des Linterarmes. Die Leistung des Biceps kann an der Schwere des Gewichts gemessen werden, das in der Hand getragen werden kann. Über die Funktionen der einzelnen Köpfe des Muskels ist noch wenig bekannt. Der kurzc Kopf übt durch seinen Verlauf, Ursprung atm Processus coracoideus und Insertion am Unterarm, speziell die Beugung des Unterarmes aus, auch ist er cs, der in der Hauptsache die Supination ausführt. Der lange Kopf, der zweigclenkig ist, wirkt nicht nur auf den Unterarm, sondern den ganzen Arm.

Wenn nicht plötzlicher Schmerz und Unfähigkeit mit dem betroffenen Arme weiter zu arbeiten den Patienten in ärztliche Behandlung bringen, so ist es oft das bauchige Hervortreten des 
muskulären Tciles des Biceps, das dem Patienten bei ciner Bicepsruptur auffällt. Die Untersuchung ergibt dann, daß der auch normalerweise sich vorwölbende muskuläre Teil des Biceps auf der betroffenen Seite stärker vortritt, mehr kugelige Form annimmt und höher oder im gegebenen Falle auch tiefer am Oberarm als der Muskelbauch auf der gesunden Seite bei der Beugung im Ellenbogengelenk sich vorwölbt. Das Abweichen dieses Muskelbauches von der normalen Lage nach der Ellenbeuge zu spricht für eine Ruptur der proximalen Schne des Caput longum, nach dem Schultergelenk zu für eine Ruptur der distalen gemeinsamen Sehne beider Köpfe. Bei vollkommenem Abreißen der langen Bicepssehne tritt häufig cin Bluterguß in mehr oder minder großem Um. fange auf, der meist im unteren Drittel des Oberarmes zutage tritt, da das Blut von den höher gelegenen Stellen herunter zu laufen pflegt. Dieser Bluterguß und die durch ihn bedingte Schwcllung können in den ersten Tagen eine richtige Diagnose unmöglich machen. Ist kein Bluterguß nachweisbar, so ist dar. nach ein vollkommener Abriß der Sehne noch nicht auszuschließen. Die Differentialdiagnose, hat nur ein Einriß der Bicepssehne oder ein vollkommener Abriß stattgefunden, ist klinisch schr schwer möglich. Wird die Sehne des langen Kopfes, die bis weit hinauf unter den Pcctoralis major sich verfolgen läßt, nicht gespannt, sondern schr schlaff und beweglich befunden, so ist cin vollkommener Abriß der Schne sehr hoch im Schultergelenk oder im Sulcus intertubercularis anzunehmen. In anderen Fällen kann man das distale Ende der abgerissenen Sehne hoch unter dem Pectoralis fühlen, wie es mir im unten angeführten Falle 3 gclang oder die Sehne liegt gar in Knäuelform auf dem vorgetriebenen Muskelbauch, wie im Falle 2. Auch in der Literatur sind Fälle beschrieben, bei denen die abgerissene Sehne, sei es in Form eines Knäuels oberhalb der muskulären Partie zu fühlon war (Kecn, R ol off, W y d le r u.a.), unter dem Pectoralis nachzuweisen war (I.oos, Fall 4), oder gar in Schleifenform mil dem Scheitel der Schleife in der Höhe des Pectoralis in peritendinösem Gewebe und perimuskulärem lindegewebe eingehüllt lag (P icard, Bazy, H of mann, Müller).

Von weitcren Symptomen ist zu crwähnen, daß der Arm nur langsam und kraftlos gebeugt werden kann. Diese Herabsetzung 
der Peugefähigkeit tritt besonders bei der Supination des Armes zutage, während in Pronationsstellung, in der der Brachialis als Beuger stärker zur Geltung kommt, das Beugen leichter möglich ist. Dieses Symptom spricht nach Hueter für eine Ruptur der langen Bicepssehne. Es ist nicht konstant nachweisbar. Wie bercits erwähnt, kann man aus der Schwerc des Gewichtes, das in der Hand bei supiniertem $\lambda$ irme getragen werden kann, ersehen, welche Arbeit der Biceps leisten kann. In den 3 von mir untersuchten Fällen von Bicepsruptur zeigte sich, daß die Patienten bei gestrecktem, supiniertem Armenicht die geringste $\Lambda$ rbeit mit dem betroffenen Arme verrichten konnten, während am gesunden die verlangte Leistung. z. B. das Heben eines Stuhles an der Lehne, ohne Anstrengung geleistct wurde. Bei Beugung des Armes im Ellenbogengelenk war dic Kraft im betroffenen Arme einmal nur wenig geschwaicht. Da bei gestrecktem, supiniertem Arme in der Hauptsache der Biceps mit Hilfe des zweigelenkigen Caput longum als Synergist mit den Schuttermuskeln und Armstreckern auf den ganzen Arm cinwirkt, während bei Beugung im Ellenbogengelenk auch die cinzclnen anderen Beuger wie Caput breve des Biceps und Brachialis mitarbeiten; so ist der $\lambda$ usfall dicser Leistungsfähigkeit als ein Anzcichen aufzufassen, daB das Caput longum des Bicepsnicht mehrzwei. gelenkig arbeitet. Das von Pagenstecher für hochsitzende Sehnenzerreißungen angegebene Symptom der Subluxation des Humeruskopfes nach vorne und oben, das zustande kommen soll, weil die Selne dann nicht mehr den Oberarmkopf gegen die Schultergelenkspfanne preßt, ist sclten nachweisbar. Es ist auch nach Ledderhose als selbständiges Symptom der Arthritis deformans, die bei Nachweis dieses Symptoms immer besteht, und nicht als Folge der Bicepsruptur anzusehen.

Lange Zeit galt in der Hauptsache das Trauma als Ursache einer Bicepsruptur. Vor allem wurde die Beanspruchung des bcreits im Kontraktionszustande befindlichen Muskcls für eine neue Leistung, der er sich dann nicht gewachsen zeigte, als Ursache für den $A b r i B$ der Schne angesehen. Die frühere Annahme, da die Ruptur des Caput longum des Biceps in der Hauptsache an der Muskelsehnongrenze vor sich gehe und daß im allgemeinen 
es sich weniger um einen $\Lambda$ briß der Sehne, als um ein Abgleiten der muskulären Bestandteile am Utbergange in die sehnigen handele, ist von Ledderhose abgelchnt worden und sind seit dieser Zeit derartige Fälle auch nicht mehr zur Beobachtung gekommen. Bei der Mehrzahl der sogenannten Bicepsrupturen handelt es sich eben um Abrisse der Sehne des Caput longum. In einzelnen Fällen, doch vicl scltener als früher angenommen wurde, ist die Schädigung rein traumatischer Natur. Als Beispicl möge ein Fall dienen, den Blencke beschrieben hat.

Ein 34j. Mann versuchte auf einen Straßenbahnwagen aufzuspringen. Es gelang ihm nicht, er wurde mit einem $\Lambda$ rme am Handgriffe des Wagens hängend mitgeschleift. Im gleichen Augenblick fuhr dic Bahn eine scharfe Kurve. Bei dem Mann entstand am Arme, an dem er sich hielt, ein typischer Bicepsriß.

Der in dem Bestreben, den Körper des schleifenden Mannes an den Wagen heranzuzichen aufs äußerste kontrahicrte Biceps, konnte der Forderung, auch die durch die Kurve bedingte erneute Beanspruchung auszuhalten, nicht mehr gerecht werden. Ma y d I spricht von der Störung der Innervierung des Muskels durch Schreck, bzw. Überraschung als Ätiologie für den Muskelriß und dieses Moment dürfte auch im vorliegenden Falle mitgewirkt haben. Nicht verständlich ist es, daß durch das Heben einer Last mit Stoß-, Schicbe- oder Wurfbewegung oder durch das Heranziehen einer Last ein $A$ briß der distalen Bicepssehne oder sonst eine Schädigung des g es und e n Muskels entstehen sollte (L o o s), es müßten doch sonst häufiger Sehnenrisse im Bereiche des Biceps bei Sportsleuten zur Beobachtung kommen, wo ja das Hochstemmen schwerster Gewichte mit Stoß- und Wurfbewegungen alltäglich durchgeführt wird. Bei den meisten dieser Bewegungen sind der Triceps und die Schultermuskulatur hauptsächlich in Anspruch genommen, doch wirkt allerdings auch der Biceps bei der dieser Bewegungen mit, wie ja überhaupt Biceps und Triceps nicht nur Antagonisten sind, sondern auch als Synergisten arbeiten (Braus).

Als rein traumatisch sind die 3 Fälle von Bicepssehnenruptur anzuführen, die L oos aufzählt, bei denen bei der Einrenkung ciner Schultergelenksluxation der Sehnenabriß eintrat.

Die Feststellung, daß die Bicepsruptur häufig bei Beanspru- 
chungen des Muskels auftrat, die nicht uber den Rahmen der alltäglichen Arbeiten hinausgingen, drängte den Gedanken auf, dal3 im gegebenen Falle die normalen Verhältnissc nicht mchr bestehen konnten. Die meisten der zur Beobachtung kommenden Fälle von Bicepsruptur betrafen Handarbeiter und ältere Männer, bei denen die Arthritis chronica als Ätiologie einer Ruptur der Sehne angesehen werden muß, worauf neben Ledderhose besonders $\mathrm{L}$ in ig e $\mathrm{r}$ cindrücklichst hingewiesen hat, der bei Tafelglasbläsern bereits zu Beginn des 3. Lebensjahrzehntes die Ruptur der langen Bicepssehne infolge chronischer Gelenkerkrankung feststellen konnte. Bei diesen Leuten war immer der linke Arm betroffen, der stärker beansprucht wird, da die schwer belastete Glaspfeife mit diesem Arm gestützt und getragen wird. Es dürfte sich erübrigen, auf die einzelnen Möglichkeiten hinzuweisen, die zu einer Bicepsruptur führen können; bei jeder Anstrengung, ja bei jeder Bewegung, bei der der Biceps innerviert wird, kann die durch die chronische Reibung über dem veränderten Knochen verdünnte Sehne durchgescheuert werden.

Zur Verth weist darauf hin, daß die spontane Zerreißung: der Sehne des langen Daumenstreckers dadurch zustande kommt, daß die Sehne dieses Muskels dauernd über die Kante des dorsalen Querbandes des Handgelenkes gleitet. Gleich dem Śchnürsenkel, der sich langsam am Schuhhaken durchscheuert, wird die Sehne langsam über den scharfen Kanten des Gelenkbandes durchgerieben. Die glcichen Vorgänge treten bei der langen Bicepssehne ein, wenn sie nicht über den glatten, mit Knorpel bedeckten Oberarmkopf gleitet, sondern sich an Rauhigkeiten und Unebenheiten im Bereiche des Schultergelenkes und Sulcus intertubercularis scheuert, die infolge chronischer Gelenkentzündung entstanden sind. Diese Knorpeldefekte oder Exostosen führen, besonders bei der I.okalisation an dem Iuberculum majus und minus, sowie in der Gegend des Sulcus intertubercularis zur Auffaserung und Verdünnung der durch das Schultergelenk und dann den Sulcus ziehenden Bicepssehne, wie Ledderhose in zahlreichen Sektionen nachweisen konnte.

Die Zerstörung der Sehne kann nun so vor sich gehen, daß sich am Rand der Sehne einzelne Fasern durchscheuern oder es kann die ganze Sehne auf einmal durchreißen. Als Vorstadium 
der Ruptur sicht Zur Verth das Stadium einer chronischen Entzündung mit kleinen Blutungen in die Sehne an und bezeichnet diesen Zustand als Tendinitis chronica. Borchers sieht in dieser Tendinitis das Anzeichen eines Regenerationsprozesses in der Sehne. Infolge dieses Entzündungszustandes in der Sehne tritt leichter ein Verwachsen 'der Schnenstümpfe nach der Ruptur mit der Limgebung ein. So berichtet Bor chers. über einen l'all, bei dem der distale Sehnenstumpf im Sulcus intertubercularis angewachsen war. Ledderhose fand bei Leichenuntersuchungen in 4 Fällen Gelenke mit Fehlen des intraartikulären Teiles der Sehne und Angewachsensein ihres oberen Sehnenendes in der Gegend des stark deformierten und obliterierten Sulcus. In allen diesen Gelenken zeigten sich deutliche Zeichen von Arthritis deformans. Bei zwei von diesen Fällen war an der Leiche die typische Dislokation des Muskelbauches des Biceps nach der Ellenbeuge zu nachweisbar, es entsprach demnach der klinische Befund dem am pathologisch-anatomischen Präparat erhobenen.

Die Ruptur der langen Bicepssehne und clic im Anschlul3 duran auftretenden Regenerationsprozesse entwickeln sich bei clen einzelnen I ällen ganz verschieden. Wirkt die chronische Reibung über dem deformierten Oberarmkopf in der Weise, dảß sich erst cinzelre Fasern der Sehne durchscheuern, so können diese Fasern bereits in der Lmgebung fest verwachsen sein, ehe der Rest der Schne sich durchgerieben hat. Das Abreißen dieser einzelnen Faserbündel bemerkt der Betroffene oft gar nicht, da es nur eine yeringe Schwächung der Kraft des Armes zur Folge hat. Ist nun der durchgescheuerte Teil der Sehne bereits fest mit den umgebenden Hüllen verwachsen, so wird auch das Durchscheuern des Restes der Schne keine größcren Ausfälle mehr bedingen. Da die abgerissenen Sehnenteile keine Spannung mehr haben, werden sie auseinanderweichen und bei günstigem Verlaufe distal und proximal der Abrilßstelle mit der Umgebung verkleben. Hat sich nun der Rest der Schne durchgerieben, so wird in diesem Augenblicke der Muskel nach der Ellenbeuge um das Stück abgleiten, um das der bereits vorher durchgescheuerte Teil der Sehne tiefer am Oberarmkopfe angewachsen ist. Ferner wird vorübergehend cin Nachlassen der Muskelkraft eintreten, da der neuc Ansatzpunkt des Muskels dem Insertionspunkt des Biceps am Unterarme näher- 
steht und der Muskel infolge der dadurch bedingten Verkürzung. den notwendigen Muskeltonus vorübergehend nicht mehr hat. In vielen Fällen sind jedoch die Erscheinungen so gering, daß sie dem Betroffenen gar nicht zu Bewußtsein kommen und erst gelegentlich einer Untersuchung an der Dislokation des Muskelbauches die Bicepsruptur erkannt wird. In dem Ausmaße der Wechselwirkungen zwischen dem Umfange der Zerstörung der Sehne und dem Grade der Selbstheilungsvorgänge durch Anwachsen der durchgescheuerten Sehnenteile an anderer Stelle, gewöhnlich im Bereiche des Sulcus intertubercularis, macht sich die eintretende Schädigung bemerkbar. Hier cin Schema aufzustellen ist nicht möglich, der Befund kann in jedem Falle verschieden sein.

Häufig fand sich, teils bei der Untersuchung, teils erst bei der Operation, daß keine Verwachsung der abgerissenen Sehne mit der Umgebung eingetreten war, sondern die Sehne lag auf dem muskulären Teil des langen Bicepskopfes zum Knäuel geballt, bzw. in Schleifenform, mit dem Scheitel der Schleife unterhalb des Sulcus intertubercularis. Die Knäuel- bzw. Schleifenbildung der Sehne möchte ich so erklären: Die Sehne steht meist im Augenblicke des Abrisses unter einer größeren Spannung, da die Sehne ja gewöhnlich bei einer Beanspruchung abreißt. Diese Spannung läßt die Sehnenstümpfe im Augenblicke der Ruptur mit einer gewissen Kraft auseinanderweichen. Je nach dem Grade der Spannung, unter dem die Sehne steht und der Weite des vorhandenen Raumes wird einfacher AbriB, Knäuel- oder Schleifenbildung der Sehne eintreten. Die Ansicht $H$ of manns, der die abgerissene Sehne mit cinem Seil vergleichen will, das an einem Ende festgehalten und geschwungen sich in Schleifenform legt, kann ich nicht teilen, da ich mir nicht denken kann, wo der zu einer solchen Bewegung notwendige Raum sein werde. Andererseits trifft dic von I,edderhose u. a. geteilte Meinung, daß die Schleifenbildung, ,das ganz allmählich eintretende Resultat der Gelenk- und Muskelbewegungen" sei, auf meinen Fall nicht zu, da der Verletzte den Arm wegen der Schmerzen sofort vollkommen schonte.

Bei Untersuchungen über die Lage der langen Bicepssehne im Senckenberg. Path. Institut Frankfurt a. M. (Direktor: Prof. 
B. Fis cher) konnte ich an der Leiche eines 68 jährigen Mannes folgenden Befund erheben.

Bci der Beugung des r. Armes der sehr muskelstarken Leiche fand sich, daß der Muskelbauch des Biceps knapp oberhalb des Ellenbogengelenks kugelig vorsprang, während sich am 1. Arm der Biceps ungefähr in der Mitte des Oberarmes nur wenig vorwölbte. Bei der Freilegung der Selhne des langen Kopfes zeigte sich, daß die Sehne bis zum unteren Ausgang des Sulc. intertub. normal verlief. Am medialen Fußpunkt des Sulcus war sie jedoch fest angewachsen. Von hier führte ein fester Strang in das Gelenk an den Ursprung der Schne. Bei der histologischen Untersuchung zeigte sich, daB dieser Strang nicht aus Sehnengewebe, sondern aus ncugebildetem Bindegewebe bestand. Im Sulc. intertub. und am Oberarmkopf fanden sich Unebenheiten, dic durch Knorpeddefekte und Knochenvorspringe bedingt waren. Aus der Anamnese des Patienten, der an eincr Apoplexic verstorben war, war nicht zu ersehen, daß er wegen dieser Biccpsruptur Beschwerden verspürt hatte.

Der Fall ist den beiden von Ledderhose anzureihen, bei denen auch der klinische Befund der Sehnenruptur mit dem pathologisch-anatomischen übercinstimmte. Die Beobachtung, daß sich die Sehne immer am medialen Teil des Sulcus intertubercularis angewachsen zeigt, ist damit zu erklären, daß die direkte Linie von der Tuberositas supraglenoidalis zum gemeinsamen Muskelbauche des Biceps mehr medial geht und daß die Biccpssehne nur durch ihren Weg durch den Sulcus intertubercularis mehr lateral, also etwas im Bogen verläuft. Sie hat demnach immer das Bestreben, diesen Bogen auszugleichen.

Im letzten Jahre kamen 3 Fälle von Ruptur der langen Bicepssehne durch indirekte Gewalt an der Chirurgischen Universitätsklinik zu Frankfurt a. M. zur Beobachtung.

I. Am 2. XII. 192 I kam der 60j. landwirtschaftliche Arbeiter Sp. in dic Klinik. Er hatte, nach seiner Angabe, am Tage vorher beim Werfen eines Heuseiles über einen bcladenen Wagen cinen plötzlichen Schmerz im $r$. Oberarm und in der Schulter verspürt. Er habe keinc Kraft mehr im Arme gehabt und deshalb dic Arbeit unterbrechen müssen.

Bei der ersten Untersuchung zeigte sich der Arm geschwollen und bei jeder Bewegung sehr schmerzhaft. Der Arm wurde für einige Tage auf eine Schiene gelcgt, bis die Schwellung zuriickgegangen war. Die erneute Untersuchung ergab jetzt: Am r. Ober- 
arm springt bei der Beugung im Eulcnbogengelenk eine kugelige Prominenz knapp oberhalb des Ellenbogengelenks vor, die etwas schmerzhaft ist und als muskulärer Teil des langen Bicepskopfes anzusehen ist. Bei Prüfung der Beweglichkeit in den Schultergelenken ergibt sich deutliche Krepitation. Auf der Röntgenplatte sind Randwucherungen an der Schultergelenkspfanne zu sehen, Symptome von chronischer Arthritis im Schultergelenk.

Es wurde nunmehr sofort eine energische Behandlung mit Massage und Bewegungsübungen eingeleitet. Bci der Nachuntersuchung nach - I Jahre - zeigte sich der r. Arm noch erheblich schwächer als der 1.; dic apfelgroße Vorwölbung oberhalb der rechten Ellenbeuge bestand noch.

Es handelte sich im vorlicgenden Falle um einen typischen Riß der langen Bicepssehne infolge arthritischer Veränderungen im Schultergelenk. Fine operative Behandlung lehnte cler Patient ab. Trotz der Arthritis chnonica glaube ich, daß bei der Operation eine besserc Funktion im Arm erreicht worden wäre.

2. Im 2. Falle handelte es sich um den 33j. Zimmermann R., der am I. II. I92 I beim Heben einer schweren Kiste einen plötzlichen starken Schmerz im r. Oberarm verspürte. R., der früher immer gesund gewesen war, mußte die Arbeit sofort aussetzen und hatte heftige Schmerzen an der Beugeseite des rechten Oberarmes.

Bei der I. Untersuchung am 2. II. war der Oberarm sichtlich geschwollen und zcigte an der Innenseitc ausgedehnte Sugillationen an der Grenze zwischen unterem und mittlerem Drittel. Beim Beugen trat knapp oberhalb des Ellenbogengelenks die kugelige Vorwölbung des herabgesunkenen langen Bicepskopfes hervor. Proximal aber nicht abgrenzbar davon fühlte man einen derberen, fast hart zu bezcichnenden, walnußgroßen, druckempfindlichen Tumor, von $\mathrm{dem}$ ein dünner Strang bis unter den Pectoralis zu verfolgen war. In bciden Schultergelenken war leichtes, vereinzeltes Knacken zu fühlen. Im Röntgenbild: war kein krankhafter Befund zu erheben. Die Kraft im r. Arme war nur ganz gering. Das Huetersche Zeichen war stark positiv. Da nach $4 \mathrm{~W}$. Massagebehandlung keine Besserung cingetreten war, wurde operatives Vorgehen beschlossen.

Der lange Kopf des Biceps wurde an der Übergangsstelle in den schnigen Teil durch cinen $15 \mathrm{~cm}$ langen Schnitt in der äuBeren Bicepsfurche freigelegt. Dabei zeigte sich, daß auf dem muskulären Teil die zu einem Knäuel zusammengeschnurte Sehne lag. Von hier führte ein derber, bindegewcbiger Strang zum Schultergelenk. Dieser Strangr wurde entfernt, die aufgerollte Sehne exstirpiert und der muskuläre Tril des äußcren Kopfes mit der Schne und dem muskulären Teil des inneren Kopfes unter starker Flexion in Ellenbogengelenk vernäht. Die Wunde verheilte glatt. Nach I $2 \mathrm{~T}$. wurde mi: Massage und Bewegungsübungen begonnen. 
Nach 1o W. war der lange Bicepskopf bereits wieder, wenn auch vorerst wenig, kontraktionsfähig, und nach $8 \mathrm{M}$. fand sich bei einer Untersuchung der lange Kopf wicder sehr gut kontraktion:s. fähig. Die Kraft und Bewegungsfähigkeit im Arme waren gegen 1 . nicht mehr hcrabgesetzt, es war vollständige Wiedcrhcrstellung eingetreten, nur fehlte die Kraft, bei gestrecktem, supiniertem Arme Gewichte zu heben, die mit dem gesunden Arme leicht gehoben werden konnten.

Trot $z$ des Fehlens von nachweisbarem Befunde am Röntgenbild und des geringen klinischen Befundes nehme ich im vorliegenden Falle an, daß es sich nicht um eine rein traumatische Sehnenruptur handelte, sondern daß arthritische Prozesse eine Schwächung der Schne bedingt hatten, weil bereits dic relativ geringe. Beanspruchung des Biceps zu cincr Ruptur geführt hatte.

Ledderhose und $L$ iniger haben ja mit anderen bereits darauf hingewiesen, daß das Fehlen sichtbarer Kennzeichen von Arthritis im Röntgenbilde das Bestehen dieser Schädigung keineswegs ausschließt. Bemerkenswert ist in diesem Falle, daß sich die Sehne zusammengeballt auf der muskulären Schicht fand und daß von dieser Sehne ein Strang zur Schultergelenkskapsel zog. Dieser Gewebsstrang wurde vom Perimysium des retrahierten muskulärerı Teiles und dem peritendinösen Gewebe des langen Kopfes gebildet. Dieses Gewebe kann, wie ich bei zahlreichen Sektionen feststeller: konnte, erhebliche Dicke annehmen, und so ist es verständlich, daß der Strang bei der Frcilegung im ersten Augenblicke für die Sehne gehalten wurde.

3. Der 3. Fall betraf einen $7 \mathrm{rj}$. Landwirt, der immer gesund war und nur in letzter Zeit über Rheumatismus im rechten Kniegelenk zu klagen hatte. Er verspürte beim Aufladen von Dickwurzblättern mit ciner Heugabel einen plötzlichen starken Schmerz im r. Oberarm, muBtc die Arbeit sofort unterbrechen und wegen der Schmerzen einen Arzt aufsuchen.

Bci der Ujtersuchung fand sich ein typischer Bicepsriß am r. Oberarm mit Schwcllung und starken Sugillationen an der Grenze zwischen mittlerem und unterem Drittel des Oberarmes. Man konnte die Sehne des langen Bicepskopfes vom muskulären Teil bis unter die Pectoralissehne verfolgen, dort fühlte man das abgerissene distale Sehnenende sich unter den Fingern bewegen. In den Gelenken, besonders aber den Schultergelenken war ausgesprochenes Krepitieren $z u$ fühlen, aber auch in diesem Falle am Röntgenbilde kein Befund zu erheben, der für Arthritis deformans 
sprechen konnte. Beim Versuch, den Arm gegen Widerstand im Ellenbogengelenk zu beugen, zeigte sich, daß die Kraft im betroffenen Arm gegen den gesunden Arm geschwächt war. Dem Patienten war es aber nicht möglich, auch nur die geringste Leistung bei supiniertem, gestrecktem Arme auszuführen. Da die Sehne vollkommen abgerissen war, wurde trotz des hohen Alters in Anbetracht der Geringfügigkeit des Eingriffs operative Behandlung beschlossen.

Nach einem $15 \mathrm{~cm}$ langen Schnitt vom Akromion bis zur Mitte des Oberarmes wurde der Sulc. intertub. freigelegt. Wie erwartet, wurdc in ihm die Sehne nicht gefunden. Sic lag aufgefasert unter dem Pectoralis. Um den Eingriff möglichst zu beschleunigen und da ja an der Ätiologie kein Zweifel bestand, wurde das Gelenk nicht weiter eröffnet. Das distale Ende wurde mit der Kornzange unter dem Pectoralis bis zum Sulc. intertub. hinaufgezogen und dort am medialen Fußpunkt des Sulcus an der Vagina mucosa intertub. und dem Periost mit einigen Seidennähten fixiert. Da es nicht gelang, den Muskcl ganz nach oben zu ziehen und so die Dislokation des Muskelbauches nach der Ellenbeuge zu ganz auszugleichen, wurde darauf nach einigen vergeblichen Bemühungen verzichtet, denn an der Wiederherstellung der anatomischen Lage konnte weniger gelegen sein, als am funktionellen Ergebnis. Es folgte Naht der einzelnen Schichten. Der Arm wurde in Pronationsstellung und in starker Flexion im Ellenbogengelenk am Körper fixiert. Die Wunde verheilte glatt. Am Io. T. wurde mit Massage angcfangen. Nach 2 M. kontrahierte sich der Biceps gut, der r. Arm war wieder kräftiger als der 1 .

Bei diesem Falle ist auffällig, daß das Huetersche Symptom nicht ausgesprochen vorhanden war. Diese Beobachtung haben auch andere Autoren bei vereinzelten Fällen gemacht. Es besteht die Möglichkeit, daß schon länger, dem Patienten unbewußl, eine Schwächung der Kraft des Armes durch teilweise Durchscheuerung der langen Bicepssehne infolge der narhgewiesenen Arthritis deformans bestand, die ein Eintreten des inneren Kopfes des Biceps und des Brachialis für die ausgefallenen Funktionen zur Folge gehabt hat, während die Funktion des Hebens mit gestrecktem, supiniertem Arme, die allein durch den langen Kopf erfolgen kann, mit dem vollkommenen Abriß dieser Sehne auffallen mußte.

Die Behandlung der Ruptur der langen Bicepssehne war früher vorwiegend eine konservative. Man versuchte durch Wickelungen die Sehne wieder ihrem Ursprungspunkte, bzw. die Sehnen- 
stümpfe wieder einander zu nähern und legte auch häufig ruhigstellende Verbände an. In neuerer Zeit ist diese Methode der Behandlung verdrängt worden durch die funktionelle Therapie mit Massage und Bewegungsübungen bald nach dem Sehnenabriß. Liniger empfiehlt wohl Aussetzen der Arbeit und das Tragen einer Mitella für einige Tage, dann aber soll der Patient wieder zuerst leichtere, dann schwerere Arbeit verrichten. Es ist in diesen Fällen, nach seiner Erfahrung, gewöhnlich das Wiedereintreten voller Erwerbsfähigkeit nach I Jahr zu erwarten. Auch andere Autoren, vor allem Ledderhose, halten die konservative Behandlung in sehr vielen Fällen für ausreichend, ohne aber ausgesprochene Gegner der operativen Bchandlung zu sein.

Bei der operativen Behandlung der Ruptur der langen Bicepssehne sind bis jetzt 3 verschiedene Methoden angewandt worden:

I. Wiederherstcllung der normalen Verhältnisse.

2. Verpflanzung des distalen Stumpfes des langen Kopfes des Muskels auf den kurzen Kopf.

3. Schaffung eines neuen Insertionspunktes für den distalen Stumpf der abgerissenen langen Sehne des Biceps.

Die direkte Vereinigung der Stümpfe der zerrissenen Selıne unternahmen v. H o c h s tet ter, $\mathrm{Hah}$ and $\mathrm{Ke}$ en. Bei v. Hoch stetter und $\mathrm{Hahn}$ war die Rißstelle in der Sehne unterhalb des Sulcus intertubercularis, bei Keen war die Sehne im Sulcus abgerissen. In dem Fall von v. Hochstetter war gemäß seinen Mitteilungen nach 9 Monaten wieder volle Funktionsfähigkeit des Armes eingetreten, während bei den beiden anderen Fällen genauere Mitteilungen fehlen. Kühner wurde in dem Falle vorgegangen, über den Bor chers berichtet. Es handelte sich um eine Sehnenruptur durch direkte Gewalt bei einem 23 jährigen Manne. Bei der Operation fanden sich im Sulcus intertubercularis Knochenvorsprünge, die von beiden Seiten den Sulcus beengten. An diesen Knochenvorsprüngen hatte sich die Sehne durchgescheuert. Die Exosten wurden abgemeißelt und die Sehnenstümpfe in dem so neu geschaffenen Sulcus wieder Seit zu Seit vereinigt. Über das Dauerresultat ist nichts bekannt, doch war die Muskulatur des Armes nachi 8 Wochen bereits sehr gekräftigt. 
Die Methode der Überpflanzung des muskulären Teiles des abgerissenen langen Kopfes des Biceps auf die Sehne und den muskulären Teil des kurzen Kopfes wurde von Bazy, Chal. mers Da Costa (zit. nach Müller), in einem von Liniger und in meinem angefuhrten zweiten Falle angewandt. In einem weiteren, von $\mathrm{M}$ ülle r veröffentlichten Falle wurde die intraartikulär abgerissene Sehne am Processus coracoideus angenäht, also auch dem langen Kopfe die Verlaufsrichtung des kurzen gegeben. Über die Endresultate ist zu berichten, daB Chalmers Da Costa nach 9 Monaten wieder gute Funktionsfähigkeit des Armes ohne Beschwerden feststellen konnte. Bei dem Falle, über den $M$ üller berichtet, bestand noch nach 6 Wochen Atrophie am Arme, genauere Mitteilungen über das Endresultat werden nicht gemacht. In dem von uns operierten Falle war nach 8 Monaten der Arm wieder kräftig, immer aber noch schwächer als der linke, die Fähigkeit, bci gestrecktem und supiniertem Arme größere Leistungen zu vollbringen fehlte. Im Falle $\mathrm{Ba} z$ y war $\mathrm{I} 3 / 4 \mathrm{Jahre}$ nach der Operation eine $\Lambda$ trophie des Transplantats cingetreten.

Roloff und Wydler hefteten das distale Ende der abgerissenen Sehne hoch oben in der Schultergelenkskapsel an, nachdem die Schne durch den Sulcus intertubercularis nach oben gezogen war. Hoffmann nähte die Sehne an die Sehne des Pectoralis major, bei I reyzehner und in dem Falle von Liniger wurde die Sehnenscheide als neuer Ansatzpunkt für die Sehne genommen. Im Falle 3 nähte ich die Sehne an den medialen unteren Ansatzpunkt des Sulcus intertubercularis an. $M$ a $B$ gebend warfür mein Handeln dervon Ledderhose und auch von mir erhobene Befund der Selbstheilung bei Bicepsruptur, beidem dieabgerissene Sehne sich an diesem Punkte einen neuen Inser. tionspunkt selbst geschaffen hatte. Dreyzehner und $\mathrm{H}$ of $\mathrm{f} \mathrm{m}$ a $\mathrm{n} n$ berichten über baldige, völlige Wiederherstellung bei ihren Patienten nach 6 bzw. I2 Wochen. In dem von mir operierten Falle hat die Kraft im betroffenen Arme wohl bald wie'der zugenommen, doch glaube ich, daß ein noch besseres Ergebnis erzielt worden wäre, wenn nicht äußere Umstände dic Durchführung einer energischen Nachbehandlung unmöglich gemacht hätten. Immerhin war nach 6 Monaten der rechte Arm wieder 
kräftiger als der linke. Während nun im Falle Wy dler nach Io Monaten die Kraft im rechten Arme gleich der im linken war und keine Muskelatrophie bestand, ril in dem Falle, den R ol of $f$ operiert hat, die Sehne nach $3 / 4$ Jahren erneut. Linigrer endlich berichtet über ein mäßig gutes Resultat, das er gelegent. lich einer Begutachtung bei einem Manne sah, dem nach einer Bicepsruptur die Sehne an der Schultergelenkskapsel angenäht worden war. Hier ist die mangelnde Funktionsfähigkeit wohl auf die zu lange durchgeführte Fixation des durch arthritische Prozesse schwer veränderten Gelenkes zurückzuführen.

In der Liste der Bicepsrupturen, die bis 1902 veröffentlicht worden sind und die von Loos zusammengestellt wurde, finden sich nur wenige Mittcilungen über das Ergebnis der Behandlung von Bicepsrupturen nach längerer Beobachtungszeit. Bei einer Zusammenstellung von 66 Fällen wird in 9 Fällen über eine erhebliche zurückgebliebene Schwäche im betroffenen Arme berichtet. Es finden sich auch bei anderen Autoren immer eine gewisse Zahl von Fällen, bei denen durch die konservative Behandlung nur geringe Besserung erzielt werden konnte. Auch in dem Falle I war der Erfolg der Behandlung nur sehr gering.

Von den Operationsmethoden ist die direkte Vereinigung der Stümpfe bei der Ruptur der Sehne oberhalb oder innerhalb des Sulcus bzw. die Anheftung der Sehne an der Tuberositas supraglenoidalis nur dann zu empfehlen, wenn es sich um rein traumatisch bedingte Sehnenabrisse handelt, sei es, daß sie durch direkte oder indirekte Gewalt entstanden sind, und wenn mit an Sicherheit grenzender Wahrscheinlichkeit das Bestehen einer chronischen Arthritis im Schultergelenk auszuschließen ist. Unter diesen Umständen ist, besonders wenn es sich nicht im Handarbeiter handelt, diese Methode die Operation der Wahl, doch dürften diese Vorbedingungen nur selten erfüllt sein. Die in dem Falle Borchers angewandte Technik der Abmeißelung der im Sulcus befindlichen Exostosen dürfte nur vereinzelt angebracht sein, da ja keine Gewähr vorhanden ist, daß sich diese Knochen. veränderungen nicht wieder bilden.

Die Überpflanzung des langen Kopfes auf den kurzen ergibt gute Ergebnisse, doch ist bei dieser Operationstechnik von vorneherein ein Verzicht auf Wicderherstellung der normalen anato- 
mischen Verhältnisse zugestanden, da der kurze Kopf viel weiter vorne ansetzt als der lange.

Der lange Kopf wird in der Folge auch nur die Leistung des kurzen ausführen können, mit dem er durch die Operation beide Insertionspunkte gemein hat.

Bei der dritten Operationsmethode, dem Anheften des distalen Sehnenstumpfes im Verlaufe der natürlichen Lage der Sehne ist in der Literatur einmal über ein Rezidiv berichtet worden. Abgesehen von der im betreffenden Falle nachgewie. senen Veränderung im Sehnengewebe selbst, scheint es überhaupt nicht günstig zu sein, den distalen Sehnenstumpf in oder oberhalb des Sulcus intertubercularis zu befestigen, denn im Bereiche des Sulcus bzw. der Tubercula treten bei der Arthritis am häufigsten Knochenveränderungen auf, so daß hier die Sehne sich leich: erneut durchscheuern kann. Bei der Anheftung am unteren Ausgange des Sulcus besteht diese Gefahr nicht. Es ist kein Anhaltspunkt dafür gegeben, daß die Fixation unterhalb des Sulcus Nachteile gegenüber einer höher gelegenen $\Delta$ nheftung der Sehne bietet, besonders da der Hauptvorteil des normalen Verlaufes der langen Sehne, der durch das Überspringen zweier Gelenke bedingt ist, auch bei dieser Technik nicht erreicht wird. Der Eingriff an sich ist sehr einfach, ein Vorteil gegenüber der ersten Operationsmethode, dic bei hoher Abrißstelle der Sehne eine Eröffnung des Gelenks erforderlich macht. Es besteht aber auch keine Indikation, die 2. Operationsmethodeder 3. vorzuziehen, die den normalenVer. hältnissenmehrentspricht und dieglcichen, wenn nicht bessere Erfolgezeitigt.

Bei allen 3 Operationsmethoden ist in der Nachbehandlung vor allem darauf $\mathrm{zu}$ achten, daß das Schultergelenk nicht $\mathrm{zu}$ lange durch einen fixierenden Verband ruhig gestellt wird, da bei den meist durch arthritische Prozesse schwer veränderten Schultergelenken leicht eine Ankylose eintritt, worauf besonders Liniger hinweist. Er führt als Beispiel einen Fall an, bei dem nach der Ruptur der Bicepssehne ein Gipsverband angelegt wurde und in der Folge sich eine Versteifung des Schultergelenks ausbildete.

Liniger hat bei seinen. Begutachtungen sehr viele Fälle 
von Ruptur der langen Bicepssehne geschen, die ohne Operation mit guter Funktion ausheilten, während er andererseits über nicht befriedigende Endresultate berichtet, bei laallen, die operiert waren. Nach den Veröffentlichungen der letzten Zeit jedoch, bei denen in systematischer Weise operatives Vorgehen mit funktioneller Therapie verbunden wurde, sind die Endresultate fast ausnahmslos gut geworden. In relativ kurzer Zeit wurde Arbeitsfähigkeit und fast völlige Erwerbsfähigkeit erziclt. Bei der konservativen Behandlung der Ruptur der langen Bicepssehne ist der Erfolg nicht sicher, andererseits aber die Anheftung der langen Bicepssehne am unteren Rande des Sulcus intertubercularis ein schr einfacher Eingriff.

Zusammenfassend möchte ich über die Therapic bei Ruptur der langen Bicepssehne sagen:

$E_{s}$ ist wesentlich festzustellen, hat cin teilweiser Einriß oder ein Abriß der langen Bicepssehne vorgelegen. Führt den Betroffenen ein allmähliches Nachlassen der Kraft des Armes zum Arzt oder hat er zufällig den herabgesunkenen Bauch des langen Bicepskopfes bemorkt, so ist eher nur ein Einriß als ein Abriß der Sehne anzunchmen. Liegen die Erscheinungen eines Traumas vor (Zcichen eines Blutergusses, heftige Schmerzen and starke Schwächung der Kraft des Armes), so muß ein Abriß der langen Bicepssehne angenommen werden. Auch der lokale Befund (́Nachweis des distalen Schnenstumpfes) kann hier dic Entscheidung bringen, in viclen Fällen kann diese Frage aber ohne Operation nicht geklärt werden.

Als Therapie für den Einriß der Sehne empfiehlt sich konservative Bchandlung mit möglichst baldiger Wiederaufnahme der Arbeit.

Bei Abriß der Sehne ist,

I. wenn gewisse Vorbedingungen erfüllt sind (jugendliches Alter, sicheres Trauma, kein Handarbeiter, keine Arthritis deformans) durch direkte Vereinigung der Stümpfe der Normalzustand wiederherzustellen;

2. wenn diese günstigen Verhältnisse nicht vorliegen, eine direkte Vereinigung der Stümpfe wegen der Rezidivgefahr nicht 
zu empfehlen. Der Betroffene verliert damit die Fähigkeit, mit gestrecktem supiniertem Arme Arbeit zu leisten, da diese durch die Überbrückung zweier Gelenke bedingte Funktion des liiceps durch den Sehnenabriß ausfällt. Die beste Funktion im Arme wird unter diesen Umständen erreicht durch die Anheftung des distalen Sehnenstumpfes am unteren medialen Ausgang des Sulcus intertubercularis.

\section{Literaturverzeichnis.}

I. B azy, Rupture du tendon de la longue portion du biceps. IBull. et Mén. de la Soc. de chir. I895, S. 156.

2. B lencke, Zur Entstehung der subkutanen Ruptur des Biceps. Münchner med. Wochenschr. I920, Nr. 17.

3. Borchers, Die Ruptur der Sehne des langen Bicepskopfes. Bruns' Beitr. 1914, 90, S. 635 .

4. Braus, Anatomic des Menschen. I. Teil. Berlin 1921, Springer.

5. Dreyzehner. Ein Fall von Zerreißung der zentralen Sehne des Musculus biceps brachii. Arch. f. klin. Chir., Bd. 65, S. 522.

6. Frohsc-Fraenkel, Handbuch der Anatomie des Menschen von Bardeleben. Abt. 2, Teil 2. Jena Igo8.

7. Gryzywa, Ein Fall von Bicepsruptur. Wiener klin. Wochenschr. 1921, Nr. 26.

8. II a hn, Zwci Falle von subkutaner Zerreißung des Musculus biceps brachii. Wiener klin. Wochenschr. I904, S. 793.

9. Hennemann, Über subkutane Bicepsruptur durch direkte Gewalt. Diss. Frankfurt a. M. $192 \mathrm{I}$.

10. Hochstetter, v., Subkutane Ruptur des langen Kopfes des Musc.

biceps brachii. Wiener klın. Wochenschr. I890, S. 399.

Ir. Hofmann, Subkutane Ausreißung der Sehne des lingen Bicepskopfes. Wiener klin. Wochenschr. I904, S. 905.

12. Huetcr, Zur Diagnose der Verletzung des Musculus biceps brachii: Arch. f. klin. Chir. 1864, Bd. 5.

13. Ledderhose, Ruptur des Biceps brachi. Deutsche Zeitschr. f. klin. Chir. 1909, Ior, S. 126.

14. Liniger Zur Fragc des typischen Bicepsrisses. Monatsschrift f. Unfallheilk. 1910, Bd. 10/11.

15. - Ein Fall von sog. Bicepsriß. Zeitschr. f. Versicherungsmed. 1913, 6, S. 65 .

16. Loos, Subkutane Bicepsruptur. Bruns' Beitr. 1901, 29, S. 410.

17. Maydl, Subkutane Muskel- und Sehnenzerreißungen. Deutsche Zci. schr. f. Chir., Bd. 17, S. 306. 
Zur Frage der Entstehung und Behandlung der subkutanen Ruptur usw. $\quad 329$

18. Mueller, Ein Beitrag zur Frage der Bicepsruptur. Bruns' Beitr. I9I 2, 78, S. 295.

19. Pagenstecher, Uber Muskel- und Sehnenabrisse am Biceps brachialis. Münchner med. Wochenschr. 1900, Nr. I7, S. 572.

20. Roloff, Abriß der langen Bicepssehne. Deutsche Zeitschr. f. Chir. I9I I, rog, S. 6I4.

2r. Schuelein, Subkutane Ruptur des Biceps brachii durch direkte Gewalt. Münchner med. Wochenschr. I9I9, Nr. 47, S. 1352.

22. Verth, zur, Über spontane Zerreißung der Sehne des langen Dau. menstreckers. Deutsche Zeitschr. f. Chir. 1909, 102, S. 569.

23. Wi es mann, Ùber einen Fall von doppelseitiger Bicepsruptur. Bruns' Beitr., Bd. 49, S. 161 .

24. Wolf, Zur Kenntnis der subkutanen Muskelrupturen und ihrer operativen Behandlung. Bruns' Beitr., Bd. 55, S. 496.

25. W y d l e r, Ein Beitrag zur Frage der Ruptur des Biceps brachii. Korrespondenzbl. f. Schweizer Ärzte 1917, S. 1793. 